\title{
Simulation Optimization of airflow level posture sensor
}

\author{
Duanlei $^{1, a^{*}, \text { Piao linhua }}{ }^{2, b}$, Dong haoliang ${ }^{3, c}$
}

(1Sensor Technique Research Center, Beijing Information Science \& Technology University, Beijing 100101,China;2 Beijing Key Laboratory for sensor, Beijing Information Science \& Technology University, Beijing 100101;3Sensor Technique Research Center, Beijing Information Science\&Technology University, Beijing 100101,China;a duanlei6688@yeah.net; b bjplh@163.com;

Keywords: airflow level posture sensor; tilt; temperature of hot source

Abstract. In this paper, using the optimization method of replacing the temperature with power to calculate the temperature distribution of the airflow level posture sensor in closed cavity. The temperature distribution of sensor in closed cavity is calculated at different inclination sensor status and under different environmental temperatures. Calculation results and experimental verification show that the two thermal resistance difference of airflow level posture sensor has good linear relationship with the inclination, and the change rate is $2.6183 \mathrm{~K} /{ }^{0}$. It has a good similarity with the experimental, and provides a theoretical basis for designing airflow level posture sensor.

Airflow level posture sensor is a new type of tilt sensor which is made by the character of the natural convection gas in the enclosed cavity. In the finite element analysis of airflow level posture sensor sensitive mechanism, the previous method use the constant temperature which is set a fixed temperature in the heating resistance device, for making the finite element calculation simple ${ }^{[1,2]}$ and theoretically explaining the working principle of the sensor. In practice, the circuit of constant temperature is more complex, difficult to implement, but the method of load a certain power has been applied widely in practice because of its simple circuit, low cost, easy to implement. This method is more actual, the error of finite element calculation is smaller, so it has more theoretical guidance significance. So this paper adopts the method of loading a certain power to optimize the finite element calculation of airflow level posture sensor, solve the temperature field and flow field through the FLUENT software, so as to explain the sensitive mechanism of airflow level posture sensor better, provides theory basis for developing airflow level posture sensor.

\section{The working principle of airflow level posture sensor}

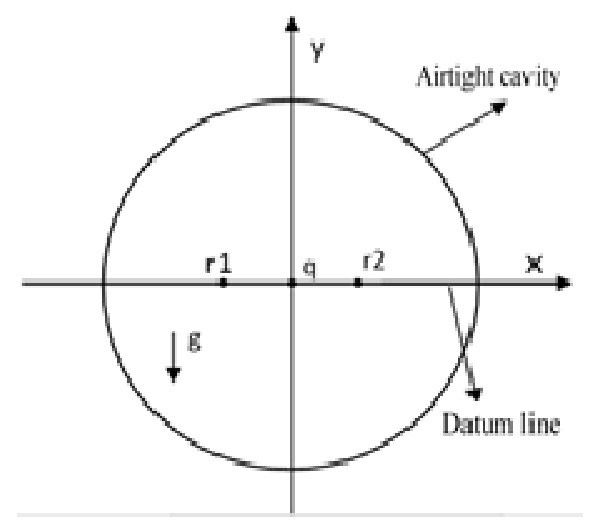

Fig.1 sensitive element simplified diagram

The sealed cavity of airflow level posture sensor is axisymmetric, the diameter of the thermal resistor is far less than the radius of the cylinder. As shown n Fig.1As shown n Fig.1, the two 
dimension structure's heat source is simplified to a circle, acceleration of gravity loads on the Y axis, sealed cavity radius $r$ is set to $10 \mathrm{~mm}$, four walls keep radiating. The resistance value of heat resistance wire is the same, two wire structure thermal resistor R1, R2 are symmetrical about the origin, the spacing of $\mathrm{d}$ is $6 \sim 10 \mathrm{~mm}$. The heat $\mathrm{q}$ of three wire structure is in the origin of the coordinate axes. By symmetry, the two thermal wires are at the same temperature, the bridge output is zero at this time. When the sensor tilts, because of the effect of natural convection, the two thermal wires are in different temperature contours, and thus lead to two different thermal wire resistance changes, finally the circuit output a certain voltage, through the output voltage we can get the tilt angle.

As shown in Fig.2, the detection circuit airflow level posture sensor is a whole bridge of Wheatstone bridge circuit, the two arm of detect bridge consists of two metal thermal resistor $\mathrm{R} 1$ and R2. The working principle is that when the sensor tilts a certain angle relative horizontal, the two thermistor position change relative in temperature field, make the two thermistor temperature one drop and another rise, the two metal thermistor resistance change, caused current change, a bridge becomes out of balance, the output $U$ is corresponding to the angle $\theta$.

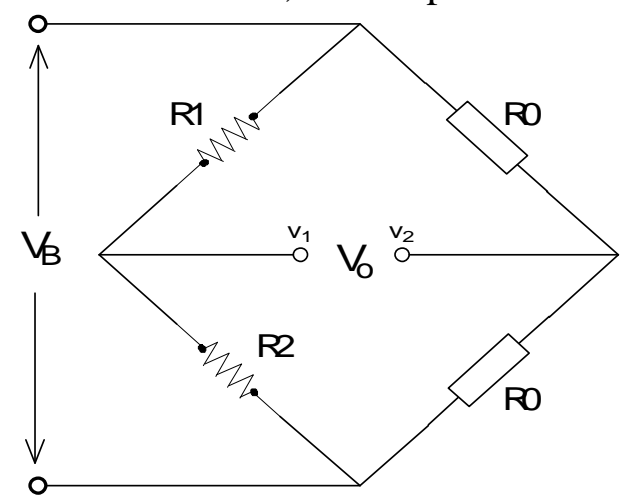

Fig.2 Measure bridge circuit principle diagram

At this point, as shown in Fig.2 the bridge output can be represented as:

$$
\begin{aligned}
& V_{0}=V_{B} \Delta \mathrm{R} / 2 \\
& \Delta \mathrm{R}=\mathrm{R} 1-\mathrm{R} 2=\alpha_{1} \mathrm{R}_{0}\left(T_{a}-T_{b}\right)
\end{aligned}
$$

Among them, the $\Delta \mathrm{R}$ is resistance difference caused by the temperature difference on both thermistor. Put the (2) into (1), the relationship between the output voltage and the temperature difference can be obtained:

$$
V_{0}=V_{B} \alpha_{1} R(0)\left(T_{a}-T_{b}\right) / 2=V_{B} \alpha_{1} R(0) \Delta \mathrm{T} / 2
$$

After the temperature field of the gas in the sensor sealed cavity is fixed, the performance of the sensor depends on the thermistor resistance differential output. Seen from (3), and two thermistor sensor output voltage is proportional to the temperature difference of values, and setting a fixed temperature on the heat source is very big difference with setting a fixed power.

\section{FLUENT Solving}

Read the .mesh file exported by the GAMBIT, before the start of the FLUENT software calculating, need to inspection the grid, and set up a series of parameters. Inspection on the grid, click the check, and check whether the minimum is negative, whether occurs any instructions of warnings or errors, this means that the mesh quality is not good enough, because grid's good and bad has great influence on computing hands no convergence, time, and the result. Then size change. Solver Settings: Solver select Pressure-based, Gravity is $9.81 \mathrm{~m} / \mathrm{s}^{2}$ set in the Y direction. Space is two dimension. Model choice: open the energy equation and laminar flow. Material Settings: set the Fluid basin is air, air properties is boussinesq, and thermal expansion coefficient is 0.003125 . The Fluid work temperature is $300 \mathrm{~K}$. Boundary condition setting: the external boundary 
type is wall, conditions is convection type, and temperature is the environment temperature of $300 \mathrm{~K}$. The internal boundary type is couple, the other keeps default. Watershed conditions set: FLUID zone keeps the default, open the SOLID setting options, select the Source Term, and set the corresponding numerical values. Solving control: Scheme of the Solution Methods set as SIMPLE, the Pressure in the Spatial Discretization set as PRESTO, other keep the default. Convergence Settings: just open the Energy and keep the default. Convergence count set as 100. At last start calculating, and display the temperature contours graphically, then read and save the temperature of the corresponding position.

\section{Calculating results and discussion}

As shown in Fig.3 is nephogram of temperature in airflow level posture sensor sensitive element under horizontal. Due to the heat source at the center of the origin, two thermal wire is symmetrical about the origin, no matter how much is the angle, the temperature field distribution is vertical upward, cloud is always the same, what change is the location of the thermal wire, the position of the thermal wire is point $a$ and point $b$.

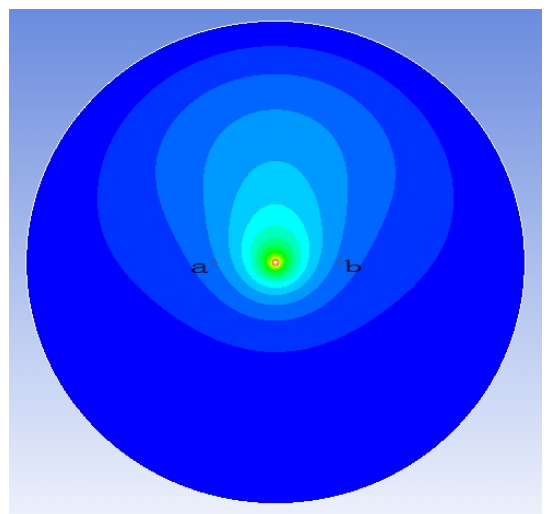

Fig.3 Nephogram of temperature in airflow level posture sensor sensitive element under horizontal

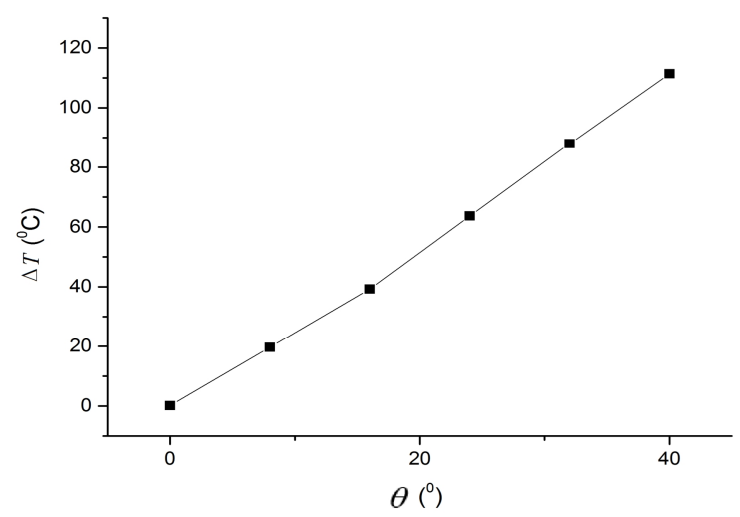

Fig. 4 The relationship curve between the temperature difference and angle of the two thermal wire 


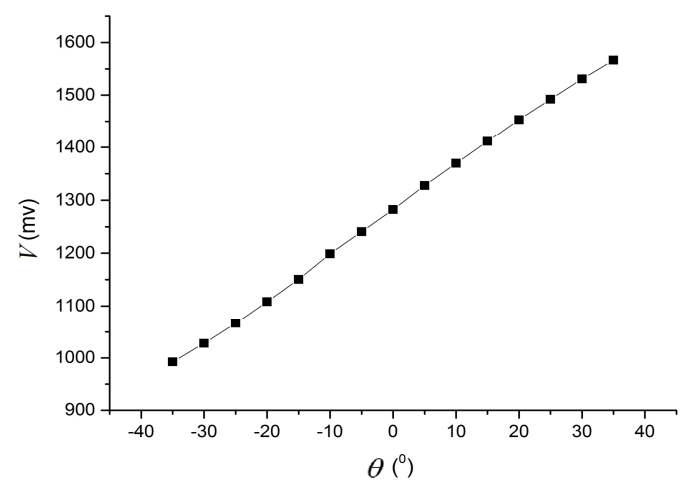

Fig.5The relationship curve between the measured output voltage and angle

As shown in Fig.4 the environmental temperature of airflow level posture sensor is $300 \mathrm{~K}$, heat source keeps a certain power, with the increase of angle, the temperature difference of two thermal wire also increase. When angle increases, the airflow keeps vertical upward, the nephogram unchanged relatively under horizontal, but the location of the thermal wire changed, one moves to the direction of the higher isothermal temperature value, another moves to the direction of the lower temperature isotherm value. Within the scope of a certain Angle, the greater the angle is, the bigger the temperature difference is, and the temperature difference has good linear relationship with angle, using matlab to calculate the linear relationship between them it is concluded that the slope is $2.6183 \mathrm{~K} /{ }^{0}$.

According to the formula $V_{0}=V_{B} \alpha_{1} R(0) \Delta \mathrm{T} / 2$, Fig.5 is the use of such sensor experimental measurement results, is similar with the simulation results, and have a good linear relationship.

\section{Conclusions}

By using FLUENT software, this paper calculates the temperature distribution in the sealed cavity, respectively calculated the temperature field distribution in the condition of different angle and different environmental temperature, the calculation results and experimental verification show that airflow level posture sensor's two thermal resistance difference has a good linear relationship with angle, the change of the slope is $2.6183 \mathrm{~K} /{ }^{0}$.

\section{Acknowledgement}

Beijing Natural Science Fund Project\&Beijing City Board of Education Science and technology key project ( KZ201511232034); Key Laboratory of Beijing open projects funded project; Modern Control Technology funded by Ministry of Education Key Laboratory;

\section{Reference}

[1] Sm Shen, The method of the finite element analysis on the problem of heat conduction-convention, calculating math, No.2, 1994: 170-182

[2] Y Lin, Lh Piao, Fx Zhang. The structure principle of omnibearing gas pendulum inclination sensor [J]. Electronic components and materials. May 2006, 25(5):19-22 